\title{
VACINAÇÃO CONTRA INFLUENZA NO ENFRENTAMENTO DA PANDEMIA DE COVID-19: RELATO DE UMA EXPERIÊNCIA E REFLEXÕES
}

\author{
VACCINATION AGAINST INFLUENZA IN TIMES OF COVID-19 PANDEMIC: REPORT OF AN EXPERIENCE AND \\ REFLECTIONS
}

\section{VACUNACIÓN CONTRA LA INFLUENZA EN EL ENFRENTAMIENTO DE LA PANDEMIA COVID-19: INFORME DE UNA EXPERIENCIA Y REFLEXIONES}

\author{
Igor Henrique Teixeira Fumagalli ${ }^{1}$, Graciano Almeida Sudré2, Silvia Matumoto ${ }^{3}$
}

\begin{abstract}
RESUMO
Objetivo: Relatar a experiência de pós-graduandos na campanha de vacinação contra a Influenza e refletir sobre os aspectos relacionados à aprendizagem que decorreram deste processo. Método: Trata-se de um estudo descritivo, do tipo relato de experiência, de dois estudantes de um Programa de Pós-Graduação em Enfermagem em Saúde Pública que participaram da primeira etapa da 22a Campanha de Vacinação contra Influenza, voltada à população com 60 anos ou mais e aos profissionais de saúde. Resultados: A participação dos pós-graduandos, na campanha de vacinação contra Influenza, que também se estabeleceu como uma das estratégias de enfrentamento da pandemia por COVID19, possibilitou um olhar crítico-reflexivo e o reconhecimento dos limites e das potencialidades do sistema local de saúde. Experiências como essas são estimuladoras pela diversidade dos cenários de prática e por propiciarem a imersão em situações-problemas reais, estratégia que possibilita a consolidação da aprendizagem. Conclusão: Destacam-se os esforços e a parceria entre ensino e serviço, o que promove maior efetividade às atividades de atenção em saúde, como também impacto à formação do estudante, por meio da qualificação profissional, oportunizando desenvolvimento de atividades com uma equipe multiprofissional.
\end{abstract}

Descritores: Infeç̧ões por Coronavirus; Vacinas contra Influenza; Colaboração Intersetorial; Práticas interdisciplinares; Aprendizagem.

\section{ABSTRACT}

Objective: To report the experience of graduate students in the Influenza vaccination campaign and reflect on the aspects learned from this process. Method: This is a descriptive study, more specifically an experience report, by two students from a Graduate Program in Nursing in Public Health, who participated in the first stage of the $22^{\text {nd }}$ Influenza Vaccination Campaign, for a population aged 60 years or more and for health professionals. Results: The participation of graduate students in the Influenza vaccination campaign, which has also been established as one of the strategies for coping with the COVID-19 pandemic, enabled a critical-reflective look upon and the recognition of the limits and possibilities of the local health system. Experiences like these stimulate and consolidate learning due to the diversity of practical scenarios and the possibility of immersion in real-life problem situations. Conclusion: The efforts and the partnership between teaching and service are highlighted, since they promote greater effectiveness in health care activities, as well as an impact on student training, through professional qualification, providing opportunities to develop activities with a multiprofessional team.

Descriptors: Coronavirus Infections; Influenza Vaccines; Intersectoral collaboration; Interdisciplinary Practice; Learning.

\section{RESUMEN}

Objetivo: Informar sobre la experiencia de los estudiantes graduados en la campaña de vacunación contra la Influenza y reflexionar sobre los aspectos relacionados con el aprendizaje que resultaron de este proceso. Método: Este es un estudio descriptivo, del tipo de informe de experiencia, de dos estudiantes de un Programa de Posgrado en Enfermería en Salud Pública, que participaron en la primera etapa de la 22a Campaña de Vacunación contra la Influenza, para una población de 60 años o más y para profesionales de la salud. Resultados: La participación de estudiantes de posgrado en la campaña de vacunación contra la Influenza, que también se ha establecido como una de las estrategias para hacer frente a la pandemia causada por COVID-19, ha permitido una mirada reflexiva y crítica y el reconocimiento de los límites y posibilidades del sistema de salud local. Experiencias como estas son estimulantes debido a la diversidad de escenarios de práctica y porque proporcionan inmersión en situaciones de problemas reales, una estrategia que permite la consolidación del aprendizaje. Conclusión: Se destacan los esfuerzos y la asociación entre la enseñanza y el servicio, lo que promueve una mayor efectividad en las actividades de atención médica, así como un impacto en la capacitación de los estudiantes, a través de la calificación profesional, brindando oportunidades para desarrollar actividades con un equipo multiprofesional.

Descriptores: Infecciones por Coronavirus; Vacunas contra la Influenza; Colaboración intersectorial; Prácticas interdisciplinarias; Aprendizaje.

'Graduado em Odontologia pela Faculdade de Odontologia de Ribeirão Preto da Universidade de São Paulo. Mestrando do Programa de Saúde Pública da Escola de Enfermagem da Universidade de São Paulo. ${ }^{2}$ Graduado em Enfermagem e Obstetrícia pela Universidade Federal de Mato Grosso. Doutorando do Programa de Pós Graduação de Enfermagem em Saúde Pública da Escola de Enfermagem de Ribeirão Preto da Universidade de São Paulo. ${ }^{3}$ Professora Associada Pública da Escola de Enfermagem de Ribeirão Preto da Universidade de São Paulo.

Como citar este artigo:

Fumagalli IHT, Sudré GA, Matumoto S. Vacinação contra influenza no enfrentamento da pandemia de covid-19: relato de uma experiência e reflexões. Revista de Enfermagem do Centro Oeste-Mineiro. 2020;10:e3790. [Access DOI: http://doi.org/10.19175/recom.v10i0.3790 


\section{INTRODUÇÃO}

A Influenza figura como um grande problema de saúde pública, podendo acarretar alta utilização dos serviços de saúde ${ }^{(1)}$. Nesse contexto, causa impactos negativos à assistência, levando à superlotação de unidades e exigindo melhor organização dos serviços de saúde, à medida que condições de trabalho precárias, nos serviços públicos, podem gerar o agravamento da doença ${ }^{(2)}$. Além de impactar na assistência à saúde, a Influenza provoca maiores gastos econômicos ${ }^{(3)}$ e desvela problemas estruturais do sistema de saúde que transitam desde a dificuldade de isolamento do paciente à necessidade de diminuição da transmissibilidade no próprio serviço. Apresenta alta mortalidade, a depender da população acometida, crianças menores de cinco anos de idade, gestantes, portadores de doenças crônicas não transmissíveis e idosos, principalmente aqueles com multimorbidades que fazem parte do grupo de risco ${ }^{(1,4)}$.

A Influenza pode ser causada pelos vírus $\mathrm{A}$, $B, C$ e $D$, destacando-se aqueles com maior potencialidade para causar pandemias pela sua grande circulação e sazonalidade, H1N1, H3N2, e vírus $\mathrm{B}^{(4)}$. Nos Estados Unidos, a mortalidade estimada supera a marca de 20.000 pessoas por ano $^{(3)}$, ao passo que, na América do Sul, entre os anos de 2013 e 2017, o diagnóstico positivo da doença e a maioria das mortes hospitalares ocorreram entre as pessoas não vacinadas ${ }^{(5)}$.

As complicações decorrentes da Influenza são classificadas de moderadas a graves e resultam do processo patológico desencadeado pelo próprio vírus ou por infecções secundárias pelo comprometimento tecidual. Dentre as complicações graves que necessitam de hospitalização, destacam-se a desidratação, encefalite, miocardite, rabdomiólise, falência renal, pneumonia e sepse ${ }^{(6)}$.

Outras condições que acometem o sistema respiratório podem assumir a mesma importância clínica, apresentar alta mortalidade, a depender da população acometida, tornar-se pandêmica e, consequentemente, acarretar alta utilização dos serviços de saúde ${ }^{(1)}$. Diante desse cenário, destaca-se a infecção viral denominada Coronavirus Disease 2019 (COVID-19), doença do coronavírus que surgiu, no final do ano de 2019, inicialmente declarada como emergência global pela Organização Mundial de Saúde (OMS), em janeiro de 2020 e, dois meses depois, passou a receber o status de pandemia ${ }^{(7)}$. Ao comparar a
Influenza e a COVID-19, as diferenças mais relevantes sinalizam a gravidade da segunda em relação à primeira, pois, além de causar mais óbitos, tem menor período de incubação, maior transmissibilidade, duração prolongada e as vacinas para imunoprevenção ainda estão em fase de desenvolvimento ${ }^{(7)}$, requisitando toda a potencialidade dos serviços de saúde para seu enfrentamento.

No Brasil, o primeiro caso de COVID-19 foi diagnosticado, no final de março de 2020, possibilitando a previsão de pico da pandemia no inverno, em concomitância aos picos de infecções por Influenza, período do ano em que aumenta a circulação de vírus respiratórios, cenário que levou o Ministério da Saúde à decisão de antecipação da vacinação contra a Influenza ${ }^{(4)}$.

Historicamente, o Programa Nacional de Imunizações incorporou a estratégia de vacinação contra a Influenza em 1999, a fim de reduzir complicações para a população e mortes decorrentes da doença, realizando campanhas anuais de vacinação contra a gripe ${ }^{(4)}$. Neste sentido, a Influenza por ser evitável pela vacinação que representa fator que pode impactar na pandemia de COVID-19, pois auxilia em um diagnóstico diferencial e diminui a procura por atendimento nas unidades de saúde e consequente superlotação, deixando os serviços disponíveis para atender a condição pandêmica.

No entanto algumas dificuldades surgiram, no cotidiano dos serviços de Atenção Básica, para cumprir a responsabilidade de imunização da população-alvo, considerando principalmente: logística de distribuição das doses nas diferentes regiões e cidades do país; condicionamento e transporte das vacinas; controle para readequar o fluxo de atendimento nas unidades de saúde, evitando aglomerações; falta de Equipamentos de Proteção Individual (EPI) aos trabalhadores da saúde; e falta de recursos humanos para cumprir ações básicas, vinculadas às campanhas.

Além disso, no Brasil, os recursos para vacinação em grande escala da população são escassos e existem grandes disparidades locorregionais. Mesmo diante dessa realidade, tornou-se imperativa a vacinação de grande parte da população, uma vez que contribui para que se evitem coinfecções virais e sobreposição dos sintomas que, por sua vez, geram dificuldades diagnósticas sobrecarregando, dessa forma, ainda mais os serviços ${ }^{(7)}$.

Para conseguir superar as adversidades citadas, o exercício da intersetorialidade é 
condição indispensável. Nesse contexto, as ações de cooperação entre uma Universidade Pública do interior do estado de São Paulo e a Secretaria Municipal de Saúde (SMS) da cidade onde está situada essa universidade possibilitaram a realização da campanha de vacinação em sua primeira fase. Ações como essas beneficiam todos os envolvidos: estudantes, com a possibilidade de construir o conhecimento a partir da realidade; profissionais, ressignificando suas práticas; e usuários dos serviços de saúde, por participarem de ações impregnadas pelo saber técnicocientífico $^{(8)}$. A interação dos estudantes com os serviços de saúde e as comunidades, além do trabalho em equipe interdisciplinar, são fundamentais para que as transformações necessárias sejam possíveis no processo de ensino-aprendizagem ${ }^{(9)}$.

A vivência de estudantes, na realidade local durante a formação, seja na participação de atividades em exercícios de saúde e/ou na comunidade, é fundamental, uma vez que proporcionam maior experiência nos diferentes cenários de práticas ${ }^{(10)}$. Integrar docentes, estudantes e profissionais dos diferentes serviços de saúde em torno de um objetivo compartilhado pode propiciar ações e serviços de melhor qualidade à população, visto vez que possibilita uma reorientação das práticas às reais necessidades de saúde, propondo reorganização da assistência. Além disso, fortalece a formação do estudante, para que atue em distintos cenários de prática, incrementando 0 processo formativo ${ }^{(11)}$, com oportunidades de efetivar aplicação na prática dos conhecimentos científicos, com foco no trabalho interprofissional.

Dessa forma, tendo em vista o caráter diferenciado da realização da referida Campanha de Vacinação contra Influenza, pelo contexto da pandemia de COVID-19, este estudo tem como objetivo relatar a experiência de pós-graduandos na campanha de vacinação contra Influenza e refletir sobre os aspectos relacionados às aprendizagens que decorreram desse processo de integração entre uma Universidade Pública do interior do estado de São Paulo e a Secretaria Municipal de Saúde (SMS) dessa cidade.

\section{MÉTODO}

Trata-se de um estudo descritivo, do tipo relato de experiência, de dois estudantes dos Programa de Pós-Graduação em Enfermagem em Saúde Pública da EERP/USP, que participaram da primeira etapa da 22a Campanha de Vacinação contra Influenza, voltado à população com 60 anos ou mais e aos profissionais de saúde, que ocorreu em março de 2020. Para essa campanha, - Ministério da Saúde instituiu como meta vacinar 90\% dos grupos elegíveis de Ribeirão Preto (RP), município situado do interior do Estado de São Paulo (SP), com uma população total de 703.293 habitantes, segundo estimativa de 2019 , realizada pelo Instituto Brasileiro de Geografia e Estatística $(\mathrm{IBGE})^{(12)}$.

Como estratégia, docentes da universidade e técnicos da SMS construíram um grupo, em um aplicativo multiplataforma de mensagem instantânea, por meio do qual compartilharam uma planilha contendo a disponibilidade dos estudantes da graduação e pós-graduação para a participação na campanha. $\mathrm{Na}$ sequência, selecionaram materiais técnicos para leitura, elaboraram uma vídeoaula com as orientações e explicações acerca dos papéis que seriam desempenhados por todos os integrantes, em cada local de vacinação, compuseram, por meio de uma escala, as equipes, viabilizaram os EPI e disponibilizaram os endereços dos serviços selecionados para receberem a contrapartida.

Nos locais de vacinação, visando à maior organização e eficácia do processo, o trabalho foi dividido em três fases, de acordo com a necessidade de recursos humanos apresentados pelos serviços: organização e triagem; registro dos dados e certificação dos critérios para vacinação; e aplicação da vacina e orientações pós-vacinação. Essa parceria permitiu conhecer, auxiliar e executar a assistência prestada durante esse período.

Na tentativa de evitar aglomerações de pessoas e atendendo a recomendação do Ministério da Saúde (MS,) para o enfrentamento da pandemia de COVID-19, os postos de aplicação da vacina foram descentralizados das unidades de saúde. Dessa forma, 32 escolas/creches/centros comunitários, pertencentes à área de abrangência das unidades de saúde, foram designados como locais de vacinação dos idosos, evitando assim o possível contato dessa população de risco com ambiente de maior circulação de pessoas e maior potencial para contaminação, uma vez que as unidades de saúde recebem casos suspeitos de possíveis infectados pelo novo coronavírus para atendimento e investigação. Esse redirecionamento de fluxo da população idosa possibilitou maior segurança aos próprios idosos e também às equipes de vacinação. Por sua vez, os profissionais de saúde, também público-alvo da 
campanha, foram direcionados para as unidades de saúde.

\section{RESULTADOS E DISCUSSÃO}

Para o enfrentamento da pandemia, podese perceber, mundialmente, uma diversidade de ações da Atenção Primária à Saúde (APS). Na Espanha, os serviços de APS foram interrompidos, e os profissionais realocados aos serviços hospitalares, acarretando em descontinuidade do tratamento e desorganização dos serviços; no Reino Unido, os serviços de APS tiveram participação na testagem sorológica, para a identificação de novos casos, na Inglaterra, por exemplo, a população passou a ser atendida de forma remota, tanto os casos suspeitos quanto os confirmados $^{(13)}$.

No Brasil, a experiência de Belo Horizonte, em Minas Gerais, consistiu em cancelar as consultas ambulatoriais e paralelamente mobilizar as equipes para a campanha de vacinação contra a Influenza. Recentemente, experiências em outros municípios relatam a mobilização das equipes para acompanhamento das condições crônicas graves ${ }^{(14)}$.

Em Sobral, município do Estado do Ceará, a imunização contra a Influenza também foi mantida e incentivada, como estratégia da APS, no enfrentamento da pandemia, assim como em todos os municípios do Brasil. Entretanto, diferentemente de outras realidades, inclusive a descrita no presente relato de experiência, os idosos foram vacinados contra a referida doença, em seus próprios domicílios: os profissionais de saúde ficavam na calçada e o procedimento era feito por meia porta ${ }^{(15)}$.

No caso da campanha de vacinação em Ribeirão Preto, SP, a situação de pandemia suscitou um serviço "orquestrado ao acaso", pelo fato de inicialmente ter ocorrido a suspensão das aulas dos estudantes do ensino infantil, fundamental e médio, que possibilitou a utilização do espaço dessas escolas, cedidos pela Secretaria de Educação. Na oportunidade, os próprios trabalhadores das escolas responsabilizaram-se por indicar alguns recursos materiais e físicos, para viabilizar a execução da campanha de vacinação, como: indicação de locais próximos a lavabos ou banheiros com pia para higienização das mãos; oferta de mesas, cadeiras para os profissionais; e disponibilização de locais de fácil e seguro acesso para população.

Durante os dias de campanha, a partir da mobilização das Unidades de Saúde da Família
(USF), das Unidades Básicas de Saúde (UBS), de creches, escolas e centros comunitários, verificouse que a aprendizagem dos pós-graduandos foi estimulada pelo processo de observação das ações de planejamento, sendo possível perceber o movimento realizado pelos serviços para o enfrentamento da pandemia e compreender o papel da vigilância em saúde nesse processo. Para a aproximação da prática nesse contexto, extrapolando a aprendizagem por meio da observação, algumas estratégias foram utilizadas, para a execução segura das intervenções, entre as quais se destacam: medidas de controle do acesso aos serviços com filas organizadas e manutenção de um distanciamento seguro; distribuição de panfletos para indicar a necessidade de utilização de medidas de controle do vírus pandêmico; oferta de máscaras aos sintomáticos respiratórios; prioridade de acesso à vacina daqueles com maior idade; vacinação até mesmo no carro daqueles com dificuldade de deambulação; e instalação de estação de vacinação em local ventilado e amplo. Com o exercício dessas ações, voltadas às habilidades psicomotoras, os estudantes conseguiram avançar no conhecimento e consolidar a aprendizagem, exercitando assim a importante tarefa de integrar teoria e prática.

Observar a intervenção possibilitou reconhecer as ações de precaução que se multiplicavam e mobilizaram fortemente a comunidade, no propósito de diminuir a sobrecarga dos serviços de saúde, o que fez com que os indivíduos demonstrassem proatividade em relação às medidas de distanciamento, à utilização de máscaras e ao comparecimento para a vacinação. A aprendizagem dos pós-graduandos, nesse momento, emergiu da possibilidade de verificar a adesão da comunidade às práticas incentivadas, por meio de ações de educação em saúde, bem como da forte influência dos meios de comunicação para a aquisição de novas práticas de proteção característica desses processos. As pessoas já estavam cientes do risco de contaminação e da importância da vacinação, tornando-as mais atentas às informações que eram compartilhadas.

Oportunizou-se, a partir da experiência, identificar $o$ impacto significativo da corresponsabilização para a aprendizagem com a comunidade, alternativa essa a ser acessada sempre que possível. Envolver a comunidade na adoção consciente das medidas de precaução quanto à Covid-19 promove mudança de comportamento e de atitudes individuais, como 
também propicia mudanças coletivas com respostas rápidas para o devido controle e não propagação da doença. Assim, ao corresponsabilizar o poder público, famílias e cidadãos, no controle coletivo e colaborativo do agravo, obtêm-se melhores resultados ${ }^{(16)}$.

Durante a experiência, era ação rotineira: compartilhar técnicas de armazenamento e controle da temperatura da caixa térmica, aspiração e manutenção da temperatura dos imunobiológicos; administração no local adequado; observação da atitude profissional quanto ao paciente; ergonomia; exercício da comunicação entre os profissionais dos diferentes turnos de trabalho; descarte correto dos materiais perfurocortantes e do lixo comum; e registro e repasse dos dados à coordenação local da SMS.

Nesse sentido, a interação entre universidade-serviço fortaleceu a formação dos estudantes, dos profissionais e ampliou os conhecimentos da comunidade. A visão fragmentada do trabalho pôde ser superada em processos semelhantes, ao direcionar o saberfazer para uma dimensão centrada na vida, não apenas em aspectos técnicos e procedimentais ${ }^{(11)}$, uma vez que revisitar as técnicas relacionadas ao processo de vacinação direcionou a aprendizagem para o campo das habilidades técnicas. No entanto a observação das atitudes, na execução do procedimento, seu posicionamento e postura acolhedora, a comunicação presente no encontro com o outro profissional, usuário e gestão local, movimentou a aprendizagem para a aquisição de habilidades relacionais e afetivas, fundamentais para o cuidado integral, centrado na pessoa, trazendo ganhos ao processo formativo na pósgraduação.

Mais que um aspecto técnico, com foco no procedimento ou possível oferta de imunização, logística do processo, contenção de uma doença em detrimento de outra ou diminuição do fluxo dos hospitais, a vacinação - mesmo com todas as medidas de proteção solicitadas pelo distanciamento social - possibilitou ainda a ampliação do olhar aos diferentes sujeitos que acessaram o serviço.

Um dos desafios apontados, para a pósgraduação stricto sensu, reside na necessidade de fortalecer a integração com os serviços de saúde, a partir de investimentos, para ampliar a compreensão sobre seu funcionamento, identificação de necessidades e construção de propostas de intervenção conjuntas. No entanto, ainda, são poucos os momentos em que essa articulação ocorre, mesmo quando considerados os programas profissionais, cujos alunos são trabalhadores oriundos da prática ${ }^{(17)}$. Estratégias como essas, simples no cotidiano das práticas, podem ser complexas ao ambiente formativo, visto que aproximar o estudante, para uma leitura mais adequada da realidade, tensiona uma abertura para que as pesquisas desenvolvidas futuramente sejam repensadas em relação ao impacto na sociedade. Outrossim, alguns pósgraduandos distanciam-se das práticas profissionais em detrimento da formação acadêmica e ações como as descritas no presente trabalho, permitindo a retomada de suas atividades profissionais ao se responsabilizarem diretamente pela saúde no contexto individual e coletivo.

Durante a experiência vivenciada, foi possível identificar a busca por vacinação de pessoas em diferentes condições de risco: inúmeros idosos com diabetes, hipertensão, problemas renais e cardiovasculares, que se apresentavam na triagem, referindo suas multimorbidades além daqueles com sinais de desidratação e desnutrição perceptíveis pelo turgor cutâneo, atrofia muscular e baixo peso. Desenvolver a visão ampliada no cuidado em saúde é uma ação capaz de produzir práticas humanizadas, ou seja, tendo a integralidade como processo construído pelo encontro no cotidiano, a humanização emerge como um produto(18).

Retomando 0 processo de ensinoaprendizagem proporcionado, a partir da campanha de vacinação, o suporte oferecido pelos docentes ocorreu em cascata, o que permitiu ampliar ainda mais o olhar e ressignificar uma simples campanha em um ambiente para o aperfeiçoamento supervisionado das habilidades psicomotoras, cognitivas e relacionais. Os docentes da EERP/USP, além de administrarem as doses, supervisionavam metodologicamente e ofertavam suporte aos estudantes da pósgraduação, para exercitarem a supervisão dos estudantes da graduação, assim, pós-graduandos e estudantes treinavam a administração dos imunobiológicos e a gestão do processo de trabalho. Dessa maneira, a oportunidade de aprendizagem dos acadêmicos da graduação também foi potencializada, assim como o exercício da docência pelos pós-graduandos. No processo de aprendizagem significativa, o ato de ensinar está atrelado ao ato de aprender, de modo que, na interação entre professores e estudantes, ocorre uma reconstrução e ampliação da 
aprendizagem, obtida no encontro com o outro, potencializada pelo diálogo, acompanhamento e problematização(19).

A potencialidade de experiências como a aqui relatada, de imersão na prática e colaboração intersetorial, é reconhecidamente explorada há anos no Brasil. Ações entre universidade e serviços de saúde, para promover a imunização, são identificadas em relatos de experiência desde a década de 1970 e, na oportunidade, envolveram docentes da Universidade Federal da Bahia durante a campanha de vacinação contra o surto de poliomielite em Salvador ${ }^{(20)}$.

A mobilização da universidade, para contribuir com ações diante do contexto crítico da saúde pública, trouxe a possibilidade de integrar as equipes de vacinação com estudantes de graduação e pós-graduação que saíram dos muros da universidade, para entrar em contato e aprender com a realidade do sistema local de saúde, olhar o mapa do território pertencente àquela unidade, conhecer a unidade de saúde e seus profissionais durante a retirada dos materiais para serem utilizados na campanha, conhecer trabalhadores de outras unidades escalados para compor aquela equipe de trabalho, dialogar sobre a realidade das distintas comunidades percorridas e suas particularidades acerca da busca pela vacina, enfim, oportunidades que propiciaram condições para que o estudante vivenciasse experiências como ser social, transformador e crítico $^{(21)}$

Experiências formativas como essa, para estudantes da pós-graduação, também, são ofertadas pela EERP/USP, durante o Programa de Aperfeiçoamento de Ensino (PAE), que vincula as necessidades de aperfeiçoamento prático e pedagógico do pós-graduando às necessidades dos estudantes de graduação, dos profissionais do serviço e usuários do Sistema Único de Saúde, possibilitando acréscimos profissionais no exercício de práticas colaborativas e do trabalho interprofissional. Transcender a prática para a qual foi escalado, colaborando com a aprendizagem coletiva, por meio da cooperação, promove o exercício de experiências transformadoras. Dessa forma, ocorre a aprendizagem significativa, ou seja, o estudante realiza conexões entre a experiência vivida e seu conhecimento prévio, transformando em significados o conteúdo experienciado ${ }^{(22)}$.

Outro fator positivo constatado durante a experiência relatada foi o público atingido pela campanha: foram imunizados $88 \%$ dos idosos da cidade $^{(23)}$, o que equivale a 68.820 doses aplicadas nesse público-alvo, chegando próximo à meta instituída pelo Ministério da Saúde que seria de $90 \%$. No caso da Influenza, o aumento do número de infectados de um ano para o outro sugere uma resistência da população em buscar a vacina, evidenciando que parte dessas pessoas somente recorrem à vacina, em períodos de grandes surtos, epidemias ou pandemias ${ }^{(24)}$. Neste relato, foi perceptível o aumento da procura da vacinação por Influenza, apesar de sua não eficácia contra à COVID-19.

O adiantamento da antiga e rotineira campanha anual de vacinação contra a Influenza pelo Ministério da Saúde ${ }^{(4)}$, além de possibilitar a imunização, auxiliou evitar a sobreposição de doenças semelhantes, quando considerados os sinais, sintomas e necessidades de intervenção. Desta forma, impactou na preservação institucional de leitos e serviços para o combate à pandemia de COVID-19 $9^{(7)}$.

A vacinação, nesse contexto, tornou-se uma verdadeira medida de prevenção individual, para promover a manutenção das instituições, representadas pelos serviços de saúde e sua escassez de recursos.

A participação dos pós-graduandos nesse processo possibilitou um olhar crítico-reflexivo e o reconhecimento dos limites e potencialidades do sistema local de saúde, possibilitando a consolidação da aprendizagem. Para Rezende e colaboradores (2015), ações intersetoriais repercutem positivamente no processo de ensinoaprendizagem, possuem maior efetividade e menor custo de execução, além de auxiliarem na execução de ações quanto às novas realidades de saúde e a distintos problemas sociais ${ }^{(25)}$.

\section{CONSIDERAÇÕES FINAIS}

Percorrendo a iniciativa, é possível visualizar uma "orquestra ao acaso" que serviu como fator de conveniência para a utilização dos espaços e serviços. A inserção da universidade nesse processo permitiu a ampliação da aprendizagem e a oportunidade de desenvolver atividades com uma equipe multiprofissional, praticando a gestão, acolhimento das demandas, diálogo com usuários e profissionais, que possibilitaram a realização da vacinação de forma mais segura e eficiente em um contexto de pandemia.

É importante destacar os esforços e a parceria entre universidade e serviço, que promoveram maior efetividade nas atividades de 
atenção à saúde, como também impacto na formação do estudante, por meio da qualificação profissional e da aprendizagem significativa, uma vez que a aprendizagem foi estimulada pelo processo de observação das ações de planejamento e pelo exercício das habilidades psicomotoras.

Interagir com a comunidade permitiu identificar a necessidade de corresponsabilização da pessoa, família e comunidade, assim como dos profissionais e universidade, ao mesmo tempo, em que a interação universidade-serviço fortalece a formação do estudante, uma vez que a prática transcende a aprendizagem e possibilita a aquisição de habilidades relacionais e afetivas, acarretando em ganhos no processo formativo na pós-graduação, ao aproximar o estudante para uma leitura mais adequada da realidade. Com isso, no desenvolvimento de uma visão ampliada ao cuidado em saúde, o estudante realiza conexões entre a experiência vivida e seu conhecimento prévio, transformando em significados os conteúdos experienciados.

Ao estabelecer parcerias com a universidade para campanha de vacinação contra a Influenza, no contexto de pandemia por COVID19, desenvolvendo ações intersetoriais, as dificuldades da SMS em cumprir a responsabilidade de imunização da populaçãoalvo puderam ser amenizadas e a meta de vacinação da população foi atingida com qualidade, permitindo ganhos para ensino, serviço e comunidade. Para os cursos da área de saúde, mesmo que por intermédio da pós-graduação, parcerias como essas precisam ser incentivadas, para que haja maior alinhamento do estudante com relação às questões que se colocam no campo das práticas, possibilitando o exercício, mesmo que inicial, do impacto social almejado com o desenvolvimento das atividades educacionais para esse nível de formação.

\section{REFERÊNCIAS}

1- Costa LMC, Merchan-Hamann E. Pandemias de Influenza e a estrutura sanitária brasileira: Breve histórico e caracterização dos cenários. Rev PanAmaz Saúde 2016;7(1):11-25. DOI: 10.5123/S2176-62232016000100002

2- Souza DB, Dall'Agnol CM. Public health emergency: Social representations among managers of a university hospital. Rev. Latino-Am Enfermagem 2013;21(4):7 telas. DOI: 10.1590/S0104-11692013000400023

3- Pérez-Rubio A, Eiros JM. Impacto económico y sanitario de la utilización de vacuna antigripal adyuvada con MF59 en población mayor de 65 años en España. Rev Esp Quimioter 2018 [cited $2020 \mathrm{Abr}$ 25];31(1):43-52. Available in: https://seq.es/wpontent/uploads/2018/02/perez 18jan2018.pdf

4- Brasil. Ministério da Saúde. 22 ${ }^{\mathrm{a}}$ Campanha Nacional de Vacinação contra a Influenza. Brasília: Ministério da Saúde; 2020.

5- El Omeiri N, Azziz-Baumgartner E, Thompson MG. Seasonal Influenza vaccine effectiveness against laboratory-confirmed Influenza hospitalizations - Latin America, 2013. Vaccine 2018;36(24):3555-66.

DOI: 10.1016/j.vaccine.2017.06.036

6- Keilman L. Seasonal Influenza (Flu). Nurs Clin North Am. 2019;54(2):227-43. DOI: 10.1016/j.cnur.2019.02.009

7. Strabelli TMV, Uip DE. COVID-19 and the heart. Arq Bras Cardiol. 2020;114(4):598-600. DOI: 10.36660/abc.20200209

8- Vendruscolo C, Ferraz F, Prado ML, Kleba ME, Reibnitz KS. Integração ensino-serviço e sua interface no contexto da reorientação da formação na saúde. Interface 2016;20(59):101525. DOI: $10.1590 / 1807-57622015.0768$

9- Gonçalves RCR, Gonçalves LG, Covre L, Lazarini WS, Dalbello-Araujo MR. Nós em rede: Vivências da parceria ensino-serviço produzidas pelo Programa de Educação pelo Trabalho para a Saúde. Interface 2015;19(supl 1):903-12. DOI: 10.1590/1807-57622014.0808

10- Faria L, Quaresma MA, Patiño RA, Siqueira R, Lamego G. Integração ensino-serviço-comunidade nos cenários de práticas na formação interdisciplinar em Saúde: Uma experiência do Programa de Educação pelo Trabalho para a Saúde (PET-Saúde) no sul da Bahia, Brasil. Interface 2018;22(67):1257-66. DOI: $10.1590 / 1807-$ 57622017.0226

11- Mendes TMC, Ferreira TLDS, Carvalho YM, Silva LG, Souza CMCL, Andrade FB. Contributions and challenges of teaching-service-community integration. Texto Contexto Enferm. 2020;29:115. DOI: $10.1590 / 1980-265 x$-tce-2018-0333

12- Instituto Brasileiro de Geografia e Estatística (IBGE). Estimativas da população residente com data de referência 10 de Julho de 2018 [citado em 15 mar 2020]. Brasília: IBGE; 2020. Disponível em:https://cidades.ibge.gov.br/brasil/sp/ribeirao -preto/panorama

13- Nedel FB. Enfrentando a COVID-19: APS forte agora mais que nunca! APS 2020;2(1):11-6. DOI: 10.14295/aps.v2i1.68 
14- Guimarães FG, Carvalho TML, Pinto RMB, Machado J. A organização da atenção Primária à Saúde de Belo Horizonte no enfrentamento da Pandemia Covid 19: Relato de experiência. APS 2020;2(2):74-2. DOI: 10.14295/aps.v2i2.128

15- Cunha MAR, Araújo Júnior DG, Cavalcante ASP, Martins AF, Sousa LA, Carvalho RC, et al. (RE)Organização da Atenção Primária à Saúde para o enfrentamento da COVID-19: Experiência de Sobral-CE. APS 2020;2(2):177-88. DOI: 10.14295/aps.v2i2.125

16- Oliveira AC, Lucas TC, Iquiapaza RA. What has the COVID-19 pandemic taught us about adopting preventive measures? Texto Contexto-Enferm. 2020;29: 1-15. DOI: 10.1590/1980-265x-tce-20200106

17- Silva LCP, Silva GTR. Contribuições e desafios do programa de pós-graduação stricto sensu em enfermagem. Rev Enferm Cent-Oeste Min. 2019;9:1-3. DOI: 10.19175/recom.v9i0.3605

18- Oliveira IC, Cutolo LRA. Integralidade: Algumas reflexões. Rev Bras Educ Méd. 2018;42(3):146-52. DOI: 10.1590/198152712015v42n3rb20170102r1

19- Lara EMO, Lima VV, Mendes JD, Ribeiro ECO, Padilha RQ. O professor nas metodologias ativas e as nuances entre ensinar e aprender: Desafios e possibilidades. Interface 2019;23:1-15. DOI: 10.1590/interface.180393

20- Costa IS, Dias ZN, Almeida MMG. Aspectos metodológicos de uma campanha de vacinação. Rev Bras Enferm. 1972;25(5):55-65. DOI: 10.1590/0034-716719720005000004

21- Freire P. Pedagogia da autonomia: Saberes necessários à prática educativa. 25a ed. Rio de Janeiro: Paz e Terra; 2002.

22- Figueredo WN, Veras RM, Silva GTR, Cardoso GMP. Práticas colaborativas nas urgências em Saúde: A interprofissionalidade do Programa PermanecerSUS, Secretaria Estadual de Saúde da Bahia, Brasil. Interface 2018;22(supl 2):1697-704. DOI: 10.1590/1807-57622017.0678

23- Guerrero N. Primeira etapa da vacinação contra a Influenza alcança $88 \%$ da cobertura esperada. Jornal USP 2020 [citado em 7 abr 2020]. Disponível em: https://jornal.usp.br/campusribeirao-preto/primeira-etapa-da-vacinacaocontra-a-influenza-alcanca-88-da-coberturaesperada/

24- Khalaf DK, Reibnitz KS, Lima MM, Correa AB, Martini JG. Teaching-service integration: Building the educational workshop in healthcare. Rev Bras Enferm. 2019;72(2):375-82. DOI: 10.1590/00347167-2018-0008
25- Rezende M, Baptista TWF, Amâncio Filho A. O legado da construção do sistema de proteção social brasileiro para a intersetorialidade. Trab Educ Saúde 2015;13(2):301-22. DOI: 10.1590/1981-7746-sip00011

Nota: O presente trabalho foi realizado com apoio da Coordenação de Aperfeiçoamento de Pessoal de Nível Superior - Brasil (CAPES) - Código de Financiamento 001

Recebido em: 20/05/2020

Aprovado em: 10/07/2020

Endereço de correspondência: Igor Henrique Teixeira Fumagalli Avenida do Café 1715, apartamento 417 - Ribeirão Preto São Paulo, Brasil.

CEP: $14050-230$ 